Europhys. Lett., 54 (3), pp. 335-341 (2001)

\title{
Flow along two dimensions of liquid pulses in foams: Experiment and theory
}

\author{
S. A. Koehler ${ }^{1}$, S. Hilgenfeldt ${ }^{1,2}$ and H. A. Stone ${ }^{1}$ \\ 1 Division of Engineering and Applied Sciences, Harvard University \\ Pierce Hall, Cambridge, MA 02138, USA \\ 2 Faculty of Applied Physics, University of Twente - P.O. Box 217, 7500 AE Enschede, \\ The Netherlands
}

(received 17 April 2000; accepted in final form 16 February 2001)

PACS. 47.55.Mh - Flows through porous media.

PACS. 82.70.Rr - Aerosols and foams.

PACS. 83.70.Hq - Heterogeneous liquids: suspensions, dispersions, emulsions, pastes, slurries, foams, block copolymers, etc..

\begin{abstract}
Experiments on foam drainage have so far only been performed in essentially one-dimensional flow geometries aligned with the direction of gravity. Here a foam-filled HeleShaw cell is used to examine pulsed drainage, which is the flow of a finite liquid volume, both along and perpendicular to the direction of gravity. An exact similarity solution to the generalized foam drainage equation exists, and an asymptotic analysis is presented to elucidate the nonlinear dynamics of the model. Good qualitative and quantitative agreement between theory and experiments on aqueous foams made with SDS surfactant is found when the nodedominated foam drainage model is applied.
\end{abstract}

Introduction. - Liquid separates the bubbles of foams and forms a continuous interconnected network. In sufficiently dry foams, almost all of the liquid resides in the channels (or Plateau borders), which are the regions between three touching bubbles, and in nodes (or vertices), which are the junctions of four channels. The liquid volume fraction $\epsilon$ is a macroscopic quantity, the ratio of liquid volume to total foam volume averaged over several bubbles, and varies spatially and temporally as liquid flows through the channels and nodes.

The flow of liquid through foams is resisted by viscous drag and driven by surface tension and (usually) gravity and is often called foam drainage [1] even in the absence of gravity (e.g., [2]). The simplest model makes an analogy to flow through porous media. In [3] we propose that foams have a permeability $k$ that depends on $\epsilon$ according to

$$
k(\epsilon)=K_{\chi} L^{2} \epsilon^{\chi},
$$

where $L$ is the length of a channel in the (monodisperse) foam, and $\chi$ and $K_{\chi}$ are dimensionless numbers that depend on the nature of the foam.

The characteristic exponent $\chi$ depends on the boundary condition at the liquid/gas interface $[4,5]$. In the no-slip model $[2,6]$, Poiseuille-type flow through the channels dominates the viscous drag and $\chi=1$. Alternatively, for the node-dominated foam drainage model [3,7], the interfaces are mobile, flow through the channels is plug-like, and the dominant viscous dissipation occurs in the nodes because of the merging and bending of the flows from four channels entering the node. Here we primarily investigate the case $\chi=1 / 2$, and refer the reader elsewhere [8-10] for the case $\chi>1 / 2$.

(c) EDP Sciences 
Using a continuity equation, the permeability (1), and the Young-Laplace law, we derived the generalized foam drainage equation for the spatial and temporal dynamics of $\epsilon$ [3],

$$
\mu \frac{\partial \epsilon}{\partial t}+K_{\chi} L^{2} \rho \boldsymbol{g} \cdot \nabla \epsilon^{\chi+1}-\frac{K_{\chi} L \gamma \delta_{\epsilon}^{1 / 2}}{2 \chi+1} \nabla^{2} \epsilon^{\chi+1 / 2}=0,
$$

where $\mu \approx 0.01 \mathrm{~g} \mathrm{~s}^{-1} \mathrm{~cm}^{-1}$ is the viscosity of the aqueous liquid in the foam, $\rho \boldsymbol{g} \approx 981 \mathrm{~g} \mathrm{~cm}^{-2} \mathrm{~s}^{-2}$ is the gravitational force per unit volume, $\gamma \approx 30 \mathrm{~g} \mathrm{~cm}^{-2}$ is the surface tension, and $\delta_{\epsilon} \approx 0.171$ is a geometric parameter [3]. While some surfactant systems show drainage according to $\chi=1[5,11]$, using SDS (sodium dodecyl sulfate) yields results in good agreement with the node-dominated limit $(\chi=1 / 2)$ when performing one-dimensional experiments in vertical tubes. For foams with bubbles of similar size to the ones used in the present study we obtain $K_{1 / 2} \approx 3.0 \times 10^{-3}[3,7]$. Consequently, there are no free parameters in this study.

Foam drainage does not only proceed in the direction of gravity, as capillary forces (isotropically) drive the liquid from wet to dry parts of the foam. An everyday example is pouring beer. Where the beer is wet, the liquid volume fraction is high, while away from the spout the foam is drier, causing an outward flow in addition to the downwards drainage. This superposition of flows can only be observed when the foam geometry allows for drainage in at least two dimensions, as studied experimentally in the present work for the first time.

Experimental setup. - We constructed a Hele-Shaw cell made of two plane parallel UVtransmitting Plexiglas plates, spaced $H=2.54 \mathrm{~cm}$ apart, about $1 \mathrm{~m}$ tall and $65 \mathrm{~cm}$ wide. The foaming liquid contains $10 \mathrm{~g} / \mathrm{l}$ of SDS in distilled water, which is well above the critical micelle concentration (CMC) [12]. A small amount of fluorescein salt $(0.25 \mathrm{~g} / \mathrm{l})$ is added to the solution, which, when exposed to UV radiation, emits green light. A continuous slow flow of $\mathrm{N}_{2}$ gas is forced through a porous glass frit inside the soap solution, filling the Hele-Shaw cell with bubbles of mean edge length $L=0.085 \mathrm{~cm}$ with a polydispersity of about $50 \%$. The foam inside the cell is continuously replenished with fresh foam from below, which rises at a velocity of about $0.26 \mathrm{~mm} / \mathrm{s}$ and this velocity is accounted for when determining the foam drainage speed. Thus at a fixed position in space the foam does not age, i.e., coarsening of bubbles (e.g., [13]) is suppressed, resulting in a uniform foam within the experimental field of view. For smaller bubbles coarsening proceeds faster, introducing a significant increase of average bubble size with height and possible coupling of coarsening with drainage [14].

In the two-dimensional pulsed drainage experiment, a volume of the soap solution is injected within one second near the center of the foam-filled cell. To maintain low liquid volume fractions $(\epsilon \lesssim 0.1)$, where the foam drainage model is applicable, the injection pulses were limited to $V_{\text {liq }} \lesssim 1 \mathrm{ml}$. For pulses smaller than $V_{\text {liq }} \lesssim 0.01 \mathrm{ml}$ the fluorescence was too weak to measure. The point of injection, known with an uncertainty of $\pm 0.2 \mathrm{~cm}$, sets the origin of the $(x, z)$ coordinate system, where the $x$-axis is horizontal and the $z$-axis is directed downwards. The origin of time is set by the end of the injection process, which is known within $\pm 1 \mathrm{~s}$.

The dynamics of $\epsilon(x, z, t)$ are determined from the fluorescence intensity $I$ recorded by a CCD camera. In one-dimensional forced-drainage experiments [7] we determined that $I$ is approximately linearly proportional to $\epsilon$, which we assume to be valid for the two-dimensional experiments here as well. The prefactor of the proportionality was determined from the ratio of the pulse volume to the integrated total intensity. It varies by $\sim 20 \%$ between different experimental runs. This uncertainty can be attributed in part to multiple light scattering within the plane of the Hele-Shaw cell. Within one experiment, the error in $\epsilon$ is much smaller.

Experimental results for pulsed drainage. - Figure 1 illustrates the dynamics of a pulse, showing the $\epsilon$-profiles along the direction of gravity $(z)$ and perpendicular to it $(x)$. As expected, gravity leads to a general downward movement of the peak in the $z$-profile, while 

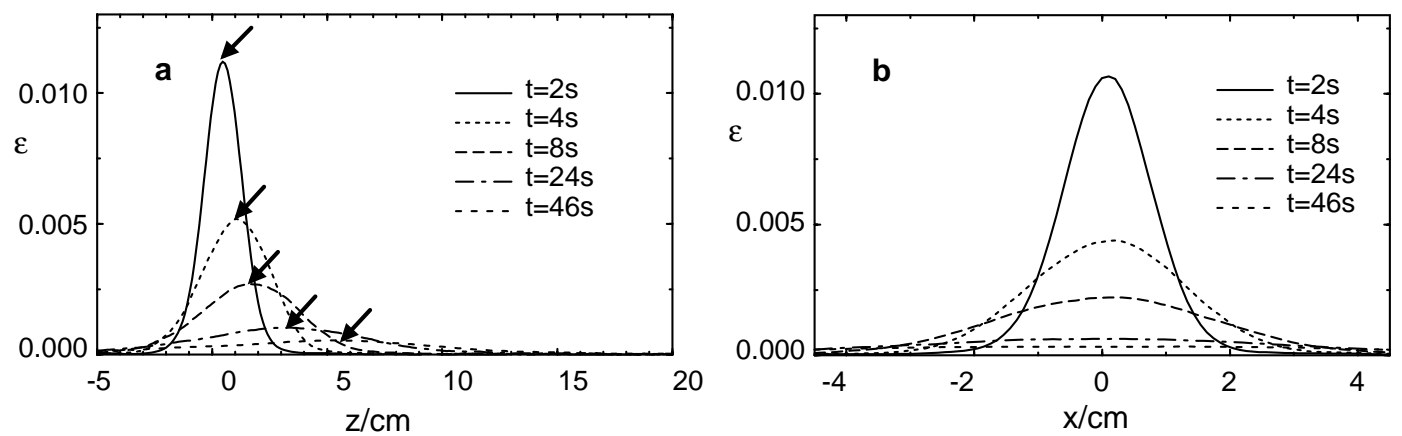

Fig. 1 - (a) Experimentally measured drainage (curves are smoothed with respect to raw data) of a $V_{\text {liq }}=0.16 \mathrm{ml}$ pulse along the vertical $z$-axis at $x=0$, and (b) along the horizontal $x$-axis at $z=0$. The arrows in (a) indicate the location of the peak, $z_{\max }$, and the peak liquid volume fraction $\epsilon_{\max }$.

the entire profile broadens along both axes due to capillarity. Within a few seconds of injection $\epsilon$ is constant across the depth of the Hele-Shaw cell (see (10) below), and afterwards the liquid volume fraction only changes in the $(x, z)$-plane of the cell.

We now discuss data from seven experiments using different pulse volumes $V_{\text {liq }}$ ranging from $0.02 \mathrm{ml}$ to $1.32 \mathrm{ml}$. For smaller $V_{\text {liq }} \lesssim 0.02 \mathrm{ml}$, the fluorescence signal is very weak and the location $z_{\max }$ and the height $\epsilon_{\max }$ of the pulse peak could not be tracked sufficiently long. Figure 2 shows that the time series are well approximated by power laws, i.e.,

$$
z_{\max } / \mathrm{cm}=c_{z}(t / \mathrm{s})^{d_{z}} \text { and } \epsilon_{\max }=c_{\epsilon}(t / \mathrm{s})^{d_{\epsilon}} .
$$

Averaging the best fits given by the solid lines in fig. 2 gives $d_{z} \approx 0.58 \pm 0.057$ and $d_{\epsilon} \approx$ $-0.90 \pm 0.045$. Note also the systematic increase of the prefactors $c_{z}$ and $c_{\epsilon}$ with $V_{\text {liq }}$.

Discussion. - During the pulsed-drainage experiment, $V_{\text {liq }}=H \int \epsilon(x, z, t) \mathrm{d} x \mathrm{~d} z$ is constant. It is convenient to non-dimensionalize this integral constraint, as well as the foam drainage equation (2), by introducing the dimensionless lengths $\xi=x / \ell_{0}, \zeta=z / \ell_{0}$, time
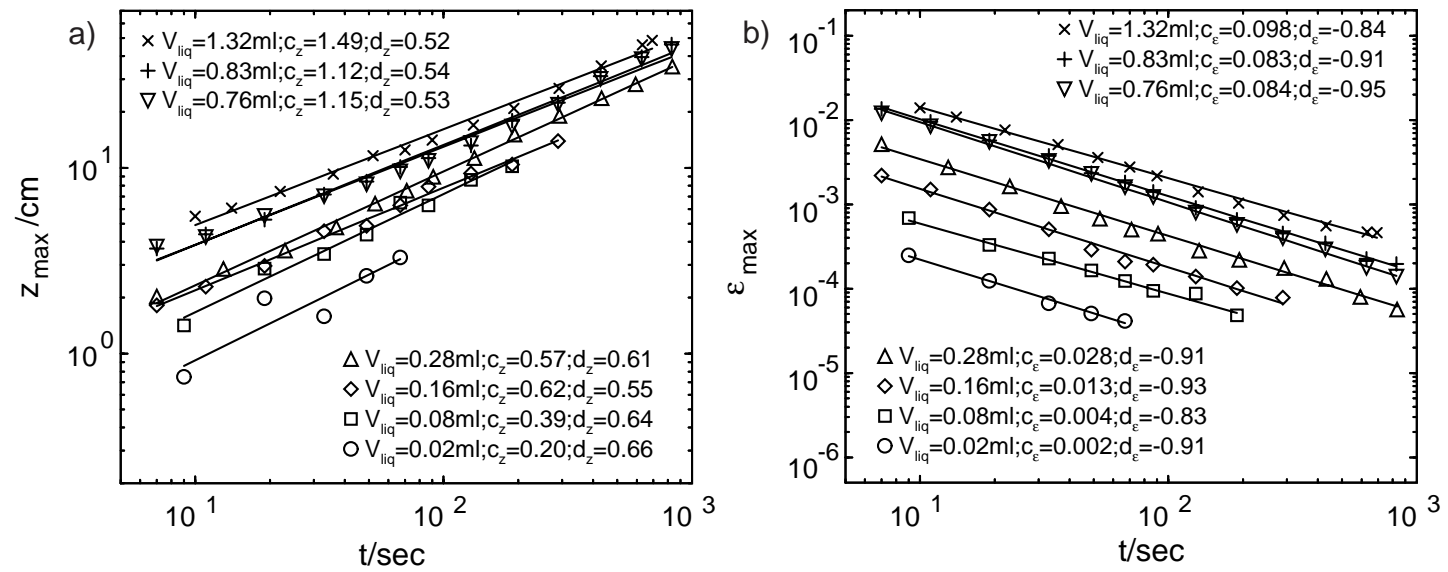

Fig. 2 - Dynamics of (a) the vertical position $z_{\max }$ and (b) the height $\epsilon_{\max }$ of the pulse maximum for seven pulses with different volumes $V_{\text {liq }}$. Solid lines are best fits to eq. (3). 
$\tau=t / t_{0}$, and a rescaled volume fraction $\tilde{\epsilon}=\epsilon / \epsilon_{0}$. Choosing the scales

$$
\ell_{0}=\frac{\gamma}{\rho g L}, \quad t_{0}=\left(\frac{2 \chi+1}{K_{\chi} \delta_{\epsilon}^{1 / 2}}\right)\left(\frac{\mu \gamma^{2 \chi} H^{\chi-1 / 2}}{(\rho g)^{2 \chi+1} V_{\mathrm{liq}}^{\chi-1 / 2} L^{2 \chi+2}}\right), \quad \epsilon_{0}=\frac{V_{\mathrm{liq}}}{H \ell_{0}^{2}},
$$

one obtains a dimensionless equation involving the characteristic exponent $\chi$,

$$
\frac{\partial \tilde{\epsilon}}{\partial \tau}+\mathcal{B} \frac{\partial \tilde{\epsilon}^{\chi+1}}{\partial \zeta}-\frac{\partial^{2} \tilde{\epsilon}^{\chi+1 / 2}}{\partial \zeta^{2}}-\frac{\partial^{2} \tilde{\epsilon}^{\chi+1 / 2}}{\partial \xi^{2}}=0, \text { with } \quad \int \tilde{\epsilon} \mathrm{d} \xi \mathrm{d} \zeta=1
$$

This equation still contains one dimensionless parameter, a Bond number

$$
\mathcal{B} \equiv\left(\frac{2 \chi+1}{\delta_{\epsilon}^{1 / 2}}\right)\left(\frac{V_{\text {liq }}^{1 / 2} \rho g L}{\gamma H^{1 / 2}}\right)=\left(\frac{2 \chi+1}{\delta_{\epsilon}^{1 / 2}}\right) \epsilon_{0}^{1 / 2},
$$

which gives the ratio of gravitational $\left(\rho g V_{\text {liq }}\right)$ and capillary $\left(V_{\text {liq }}^{1 / 2} H^{1 / 2} \gamma / L\right)$ forces.

Self-similar solution. - Equation (5) has a self-similar solution

$$
\tilde{\epsilon}(\xi, \zeta, \tau)=\tau^{-2 /(2 \chi+1)} g(q, s), \quad \text { with } \quad q=\tau^{-1 /(2 \chi+1)} \xi, \quad s=\tau^{-1 /(2 \chi+1)} \zeta,
$$

which reduces (5) to a partial differential equation in similarity coordinates $q, s$ :

$$
-\frac{1}{2 \chi+1}\left(\frac{\partial(q g)}{\partial q}+\frac{\partial(s g)}{\partial s}\right)+\mathcal{B} \frac{\partial g^{\chi+1}}{\partial s}-\left(\frac{\partial^{2} g^{\chi+1 / 2}}{\partial q^{2}}+\frac{\partial^{2} g^{\chi+1 / 2}}{\partial s^{2}}\right)=0 .
$$

Although this self-similar ansatz works for the foam drainage equation in arbitrary dimension $d$, only for $d=2$ does the volume remain conserved because $\int \tilde{\epsilon}(\boldsymbol{\xi}, t) \mathrm{d}^{d} \xi \propto t^{(2-d) /(2 \chi+1)}$, where $\boldsymbol{\xi}$ is the generalized vector of non-dimensional spatial coordinates in $d$ dimensions.

Both the foam drainage equation (5) and its self-similar version (8) can be solved numerically by an operator-splitting algorithm PDE solver. These calculations assume a perfectly dry background foam into which the pulse is introduced, so that the boundary conditions are $\tilde{\epsilon}=g=0$ far away from the pulse.

Experimental and theoretical scaling. - Beyond numerical simulations, a number of analytical results can be obtained and compared to experiments. Given the self-similar ansatz (7), we expect the position and corresponding maximal liquid volume fraction along $z$ to obey

$$
z_{\max }=c_{z} t^{1 /(2 \chi+1)} \quad \text { and } \quad \epsilon_{\max }=c_{\epsilon} t^{-2 /(2 \chi+1)} .
$$

Previous one-dimensional drainage experiments for aqueous SDS-based foams [3,7] showed that the node-dominated theory with $\chi=1 / 2$ is a good description of the data. Comparison of (3) and (9) then predicts $d_{z}=1 / 2$ and $d_{\epsilon}=-1$, in good agreement with the present two-dimensional experimental results (see fig. 2). The slight discrepancies may be due to the presence of a non-vanishing background liquid volume fraction $\epsilon_{\mathrm{b}}$ as the foam into which the pulse is introduced is not perfectly dry: Due to gravity, $\epsilon_{\mathrm{b}}$ must increase with $z$, so the drainage dynamics is accelerated, as observed experimentally.

The prefactors $c_{z}$ and $c_{\epsilon}$ in (3) and (9) depend on $V_{\text {liq }}$ and material parameters. Below, we present an asymptotic theory for analytical predictions of these prefactors.

No gravity. - If $\mathcal{B}=0$ and $\chi=1 / 2,(2)$ reduces to a diffusion equation with a diffusivity

$$
D=K_{1 / 2} L \gamma \delta_{\epsilon}^{1 / 2} / 2 \mu \text {. }
$$

For our experiments, $D \approx 0.16 \mathrm{~cm}^{2} \mathrm{~s}^{-1}$. We thus estimate the time required for a pulse to spread the thickness $H$ of the Hele-Shaw cell, and so the onset of two-dimensional spread- 

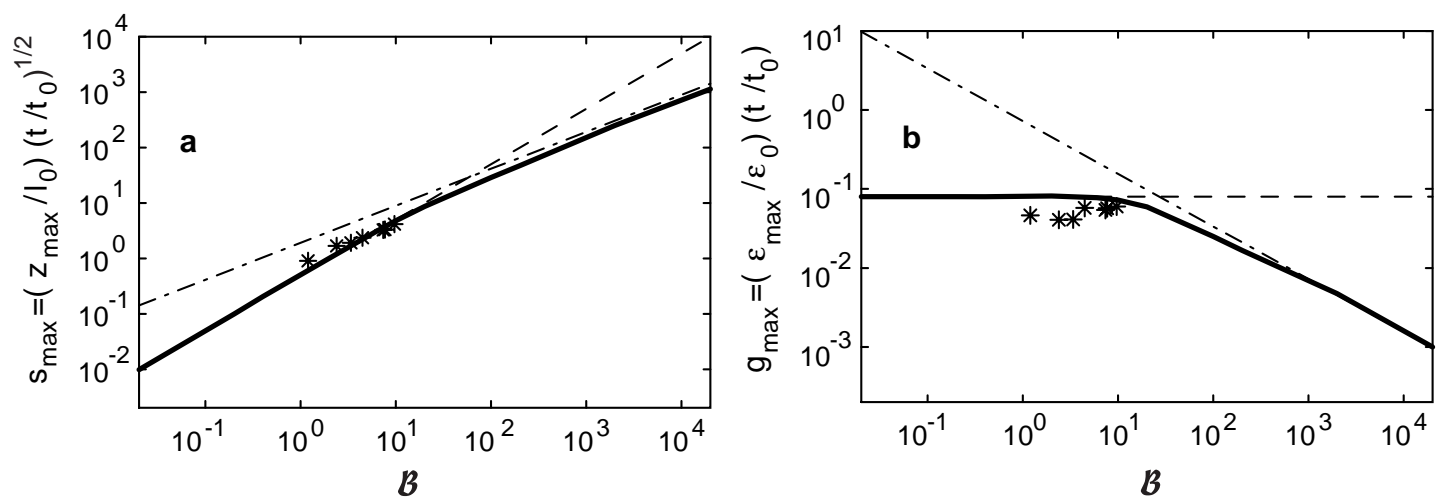

Fig. 3 - Dependence of the peak position (a) and the peak height (b) on the Bond number, plotted in self-similar coordinates. Experimental data (stars) are compared with numerical simulations (solid lines) and analytical results for small $\mathcal{B} \lesssim 10$ (dashed lines) and large $\mathcal{B}$ (dot-dashed lines).

ing, to be approximately $10 \mathrm{~s}$, so the use of data for $t \gtrsim 10 \mathrm{~s}$ in fig. 2 should be justified. A unit-volume solution to (8) with $\mathcal{B}=0, \chi=1 / 2$ is the Gaussian $g_{0}(q, s)=\frac{1}{4 \pi} \exp \left[-\left(s^{2}+q^{2}\right) / 4\right]$ which does not have compact support, unlike gravity-free solutions for $\chi>1 / 2[8,9]$.

Small B. - We now perform a perturbation expansion $g(q, s)=g_{0}(r)+\mathcal{B} g_{1}(q, s)+\mathcal{O}\left(\mathcal{B}^{2}\right)$ in (8) for small $\mathcal{B}$, i.e., for small $V_{\text {liq }}$ (we have defined $r^{2}=s^{2}+q^{2}$ ). To first order in $\mathcal{B}$ the ansatz $g_{1}(q, s)=s \exp \left(-3 r^{2} / 8\right) f_{1}(r)$ gives the linear ODE

$$
3 r\left(1-r^{2} / 8\right) f_{1}+2\left(r^{2}-3\right) \partial_{r} f_{1}-2 r \partial_{r}^{2} f_{1}=3 r /\left(16 \pi^{3 / 2}\right) .
$$

An examination of the large- $r$ asymptotics of the homogeneous equation reveals that both solutions are divergent, and therefore unphysical. Thus, the coefficients of the homogeneous solutions vanish, and we determine the particular solution of (11) by a polynomial expansion of $f_{1}$ around $r=0$. It converges onto a unique solution, whose zeroth-order (constant) part is numerically found to be $f_{1,0}=0.019758 \ldots$ In order to determine the location $s_{\max }$ and height $g_{\max }$ of the pulse peak, we demand $\partial_{s}\left(g_{0}+\mathcal{B} g_{1}\right)=0$ at $s_{\max }$ and so find

$$
s_{\max }=8 \pi f_{1,0} \mathcal{B} \approx 0.4966 \mathcal{B} \quad(\mathcal{B} \ll 1) .
$$

In fig. 3a, we compare the results of this asymptotic formula to simulations of (8), as well as to the measurements. The simulations reproduce (12) very closely, even up to $\mathcal{B} \lesssim 10$. For consistent display of the data in fig. 3 , the fits of fig. 2 were replaced by best fits to the theoretically predicted behavior $z_{\max } \propto t^{1 / 2}, \epsilon_{\max } \propto t^{-1}$. Although the experimental data span almost two decades of pulse volume, the corresponding Bond number range is $1.2 \leq \mathcal{B} \leq 9.8$, see (6). In this range, the data compare favorably to the theoretical results.

With the result $s_{\max } \propto \mathcal{B}$ from the expansion above, $g_{\max }=g_{0, \max }+\mathcal{O}\left(\mathcal{B}^{2}\right)$ follows, so the peak maximum value stays constant $\left(g_{0, \max }=1 / 4 \pi\right)$ for small $\mathcal{B}$. The dashed line in fig. $3 \mathrm{~b}$ represents this prediction, which coincides with the PDE solution (solid line). The rescaled experimental data for $g_{\max }$ again compare favorably with theory.

Large $\mathcal{B}$. - For $\mathcal{B} \gg 1$ (large $V_{\text {liq }}$ ), the advection term dominates the drainage process along the $s$-axis, and we can neglect the terms $\partial_{\zeta}^{2} \tilde{\epsilon}$ and $\partial_{s}^{2} g$ in (5) and (8), respectively. This truncation introduces an error of $\mathcal{O}\left(1 / s^{2}\right)$, which is small, because most of the liquid resides at large $s$ (the pulse asymmetry is large). The ansatz $g(q, s)=s^{\alpha} F(q)$ (i.e., a separation of variables) in the truncated version of (8) yields $\alpha=2$ and the ODE 

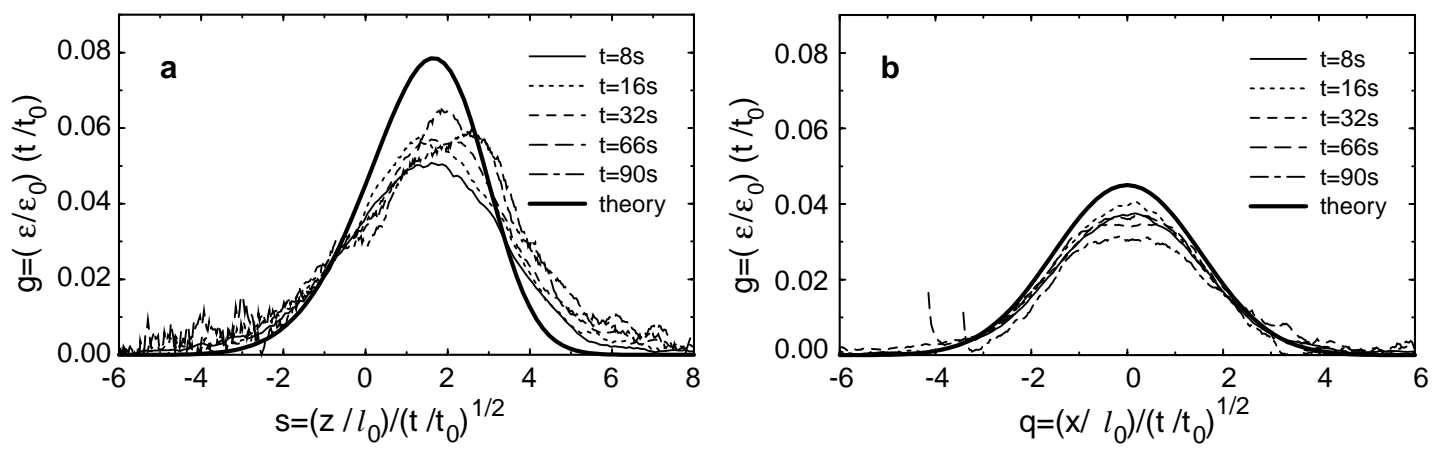

Fig. 4 - Collapse of the vertical (a) and horizontal (b) profiles from fig. 1 when rescaled according to (7). A PDE simulation with the corresponding Bond number $\mathcal{B}=3.37$ is shown as a heavy line.

$$
-2 F-\frac{1}{2} q \partial_{q} F+3 \mathcal{B} F^{3 / 2}-\partial_{q}^{2} F=0 .
$$

For symmetry reasons, $F(q)$ must be an even function, which we expand for small $q, F(q) \approx$ $F_{0}+F_{2} q^{2}+F_{4} q^{4}+\ldots$ We thus approximate the actual pulse shape by a surface in $(g, q, s)$ space with a $s^{2}$ profile at $q=0$ and a quartic profile laterally. Solving for the leadingorder coefficients of the expansion in $q^{2}$, one obtains $F_{0}=16 /\left(81 \mathcal{B}^{2}\right), F_{2}=-16 /\left(243 \mathcal{B}^{2}\right)$, $F_{4}=-4 /\left(729 \mathcal{B}^{2}\right)$. To calculate the coordinate $s_{\max }$ of the pulse maximum (which also gives the finite length of the pulse along $s$ ), we use the condition of volume conservation, namely $1=\int_{0}^{s_{\max }} \int_{-q_{0}(s)}^{+q_{0}(s)} g(q, s) \mathrm{d} q \mathrm{~d} s$, with $\pm q_{0}(s)$ fulfilling $g\left( \pm q_{0}(s), s\right)=0$. We eventually find

$$
s_{\max }=\frac{3^{11 / 6}}{4 \sqrt{2}}(\sqrt{2}-1)^{1 / 6}\left(\frac{5}{4-\sqrt{2}}\right)^{1 / 3} \mathcal{B}^{2 / 3} \approx 1.9116 \mathcal{B}^{2 / 3} \quad(\mathcal{B} \gg 1) .
$$

Figure 3 a shows that simulations for large $\mathcal{B}$ are in excellent agreement with (14). Note that the maximum experimental injection volumes $(\mathcal{B} \lesssim 10)$ are too small to reach this scaling regime. Figure $3 \mathrm{~b}$ shows the pulse height in rescaled coordinates, which consequently becomes

$$
g_{\max }=F_{0} s_{\max }^{2} \approx 0.7218 \mathcal{B}^{-2 / 3} \quad(\mathcal{B} \gg 1) .
$$

Rescaled profiles. - Apart from reproducing the pulse peak dynamics, the self-similar rescaling (7) should collapse the entire profile for a given $\mathcal{B}$ onto one master curve. Figure 4 displays this collapse for the experimental pulse data from fig. $1\left(V_{\text {liq }}=0.16 \mathrm{ml}\right)$ in $(g, q, s)$ space. Unlike $1 \mathrm{D}$ pulsed drainage, the spreading of the $2 \mathrm{D}$ pulse is diffusive, and both the horizontal and vertical pulse widths scale with $t^{1 / 2}$. A simulation of (8) for the corresponding Bond number $\mathcal{B}=3.37$ yields theoretical curves in good agreement with the data (note that the theory has no free parameters once $\chi$ is specified). We attribute the remaining discrepancies to uncertainties in the intensity calibration and/or small deviations from $\chi=1 / 2$.

Conclusions. - While we know of one earlier numerical study of $2 \mathrm{D}$ drainage [15], our work provides the first comparison of experimental and theoretical results for drainage in more than one dimension. Quantitative measurements of 2D pulsed-drainage dynamics are performed for hundreds of seconds and agree with the predictions of the node-dominated drainage model. The spreading of the pulse in both directions is diffusive and scales with $t^{1 / 2}$. In one-dimensional pulsed drainage, the pulse also spreads with $t^{1 / 2}$ for the channeldominated model $(\chi=1)$ whereas for the node-dominated model $(\chi=1 / 2)$ the pulse spreads with $t^{2 / 3}[3,9]$. 
For the two-dimensional generalized foam drainage equation (2) there is an exact volumeconserving self-similar ansatz governed by one parameter, the Bond number $\mathcal{B}$, which determines the degree of asymmetry of the pulse shape. This property is unique to 2D: in other dimensions, self-similar solutions to the PDE do not preserve the pulse's liquid content [3,9]. In the absence of coarsening, any pulse of liquid in this $2 \mathrm{D}$ geometry eventually evolves in the manner described here; however initial distributions in $\epsilon$ very different from the self-similar shape take longer to show scaling behavior.

The generalization of the present analysis to three dimensions is straightforward. In order to obtain liquid volume fraction data for 3D foam drainage, X-ray scattering (CAT scan) could provide fast data acquisition and would supplement studies using MRI [16]. The present work is valuable for the study of the dynamics of localized perturbations in the liquid content of an otherwise uniform foam, which move and spread much like the pulses investigated here.

$$
* * *
$$

We thank A. G. Evans for support through the MURI project on Ultralight Metal Structures, and M. BREnNer who wrote the original version of the PDE solver. Support by the ACS-PRF is acknowledged.

\section{REFERENCES}

[1] Leonard R. A. and Lemlich R., A.I.Ch.E. J., 11 (1965) 18.

[2] Verbist G., Weaire D. and Kraynik A., J. Phys. Condens. Matter, 8 (1996) 3715.

[3] Koehler S. A., Hilgenfeldt S. and Stone H. A., Langmuir, 16 (2000) 6327.

[4] Wilde P. J. et al., The Role of interfacial structure and composition on foam drainage and fluid dynamics, in Foams and Films: Proceedings of the International Workshop on Foams and Films, Leuven, Belgium, edited by D. WeaIRE and J. Banhart (MIT-Verlag, Bremen) 1999.

[5] Durand M., Martinoty G. and Langevin D., Phys. Rev. E, 60 (1999) R6307.

[6] Goldfarb I. I., Kann K. B. and Shreiber I. R., Fluid Dynamics 23 (1988) 244.

[7] Koehler S. A., Hilgenfeldt S. and Stone H. A., Phys. Rev. Lett., 82 (1999) 4232.

[8] Grundy R. E., IMA J. Appl. Math., 31 (1983) 121.

[9] Koehler S. A., Stone H. A., Brenner M. P. and Eggers J., Phys. Rev. E, 58 (1998) 2097.

[10] Hilgenfeldt S., Koenler S. A. and Stone H. A., Analysis of pulsed drainage in a HeleShaw cell, in Proceedings of the 3rd EuroConference on Foams, Emulsions and Applications, Delft (2000), edited by P. Zitha, J. Banhart and G. Verbist (MIT-Verlag, Bremen) 2000.

[11] Koehler S. A., unpublished data; Hutzler S., private communication.

[12] Prud'homme R. K. and WarR G. G., Foams in mineral flotation and separation processes, in Foams, edited by R. K. Prud'homme and S. A. Khan (Marcel Dekker, New York) 1996.

[13] Durian D. J., Weitz D. A. and Pine D. J., Phys. Rev. A, 44 (1991) R7902.

[14] Hutzler S. and Weaire D., Philos. Mag. Lett., 80 (2000) 419; Saint-Jalmes A., Vera M. U. and Durian D. J., Europhys. Lett., 50 (2000) 695; Hilgenfeldt S., Koehler S. A. and Stone H. A. under review.

[15] Neethling S. and Cilliers J. J., Visualization and drainage of coalescing, flowing foams, in Foams and Films (ref. [4]).

[16] McCarthy M. J., A.I.Ch.E. J., 36 (1990) 287; Gonatas C. P., Leigh J. S., Yodh A. G., Glazier J. A. and Prause B., Phys. Rev. Lett., 75 (1995) 573. 